\section{P195 Alcoholic Beverage Consumption and Associations With Calorie Intake, Body Mass Index, and Obesity in the US Population}

Francis Tayie,PhD, MPhil, francis.tayie@gmail.com, Central Michigan University, 1516 Stoddard Street, Cape Girardeau, MI 63701; R. Nelson, BS; G. Beck, BS

Objective: Number of alcoholic drinks per day and associations with calorie intake, and risks of overweight and obesity were studied.

Design, Setting and Participants: Multiple regression models were used to analyze the alcohol behavior data from the U.S. National Health and Nutrition Examination Surveys 2007-2010. Data from 7,500 participants, 3,642 men, and 3,858 women, ages $20-70$ years, were included in this study.

Outcome Measures and Analysis: Outcome variables were calorie intake, BMI, and likelihood of overweight or obesity. Potential confounding variables controlled in regression analyses were age, education, income, physical activity, marital status, smoking status, and race/ ethnicity.

Results: The proportion of this sample who had at least 12 alcoholic drinks per year was $78.3 \%$. The average daily quantity of alcohol consumed by men drinkers was about $28.7 \mathrm{~g}$ (range, 11.2-52.5 g) and by women drinkers was about $14.9 \mathrm{~g}$ (range, 7.1-41.5 g). Compared to nondrinking counterparts, alcohol consumption associated with increases in daily calorie intake, men: $243.7 \mathrm{kcal}, \mathrm{p}<0.001$; women: $135.5 \mathrm{kcal}, \mathrm{p}<0.001$. Men who had $\geq 4$ drinks daily associated with significantly higher $\mathrm{BMI}, \mathrm{P}=0.024$. Men who had $\geq 2$ drinks daily were more likely to be overweight, $\mathrm{p}=0.010$, or obese, $\mathrm{p}=0.037$. Women at all levels of drinking associated with significant decreases in BMI, $\mathrm{p} \leq 0.023$, and lower risk of overweight and obesity.

Conclusions and Implications: Alcohol consumption associates with increased calorie intake among both men and women, but only men associate with significant likelihood of overweight and obesity. Health professionals should enquire about alcohol consumption when interacting with overweight or obese men, and women who have low BMI.

Funding: None.

\section{P196 Needs Assessment for South Asian Adults Living in Texas for Developing a Culturally- Appropriate Nutrition Education Program} Zubaida Qamar, MS, qamaz25@email.tamu.edu, Texas A\&M University, 214B Cater-Mattil, College Station, TX 77840; Y. Hernandez-Garbanzo, PhD; R. Misra, PhD, West Virginia University

Objective: To assess the level of acculturation, barriers, motivators and behaviors related to the eating patterns of South Asians (SA) living in Texas.

Design, Setting and Participants: Nutrition education programs are needed for the SA population in the U.S. Their acculturation into the westernized lifestyle and genetic susceptibility of chronic diseases increase their risk for malnutrition. Cross-sectional study of SA adults $(n=105 ;$ men $=58$, women $=47$, mean age $=24$ years $)$, who completed a self-report questionnaire measuring demographics, family history of diseases, acculturation, perceived barriers and motivators from the Health Belief Model and dietary behavior items adapted from the Health Promotion Lifestyle Profile II.

Outcome Measures and Analysis: Descriptive statistics were obtained for each of the variables of this study. Also, a score for dietary behavior was determined and Multivariate Linear Regression Analysis was conducted to predict the relationship between dietary behaviors and other variables of this study.

Results: $76 \%$ reported having Family History for Diabetes, Cancer and Cardiovascular diseases. In terms of acculturation, $\sim 94 \%$ participants indicated consuming traditional SA foods at least once daily, however majority ( $~ 54 \%)$ indicated gaining weight after relocation to the U.S. The most common perceived barrier to eating healthier was "lack of time" and the most common motivator was "disease prevention". Frequency of consumption of traditional foods was the variable that significantly predicted healthy dietary behaviors $(\mathrm{p}=0.001)$.

Conclusions and Implications: Results from this study suggest a need for nutrition education among SA and will guide the development of culturally appropriate nutrition education strategies to promote healthier lifestyle and disease prevention.

Funding: None.

\section{P197 The Family Mealtime Study: Understanding Occurrences During and Surrounding Family Mealtimes}

Car Mun Kok, MS, carmun@huskers.unl.edu, University of Nebraska-Lincoln, 1400 R Street, 135 Mabel Lee Hall, Lincoln, NE 68588

Objective: Research showed that family mealtimes (FM) positively impact youth's dietary behaviors. However, the process is unclear. Understanding the processes can clarify how FM impact youth. This knowledge can be used to design better educational programming for families - a step towards obesity prevention. This mixed methods study aims to identify occurrences around FM that impact youths' dietary behaviors to promote positive FM with the goal of obesity prevention.

Design, Setting and Participants: One hundred parent-child dyads completed surveys. A subsample of 40 families participated in mealtime observations. Twenty parents were interviewed. Data collection was conducted in the participants' homes.

Outcome Measures and Analysis: Parent-child dietary behaviors, family mealtime practices, mealtime-related occurrences, and thoughts about family mealtimes were analyzed using SPSS and NVivo.

Results: Correlations showed that youth who had frequent $\mathrm{FM}(77 \%, \geq 5$ times/week) and healthy food at home had healthier food preferences. Parents who 